\title{
Magnetization profiles of AC type-II superconducting wires exposed to DC magnetic fields
}

\author{
B. C. Robert, and H. S. Ruiz
}

\begin{abstract}
The macroscopic electromagnetic behaviour of a type-II superconducting wire for alternating current power transmission under constant magnetic field conditions is captured by the numerical solution of the Maxwell equations under the framework of the critical state principle and the so-called integral formulation, also known as J-formulation. Time-dependent distributions for the flux front profiles of local current density, magnetic flux, and cycles of magnetic moment are presented. We have found that, regardless of the intensity of the applied magnetic field, the first cycle of $I_{t r}(t)$ defines the period of magnetic stabilization of the SC wire where two plateaus with constant magnetic moment can be measured. Then, a cyclic monotonic behaviour with well-defined flux-front boundaries has been identified, with clear signatures of current-like and field-like flux front profiles. This observation has allowed us to establish semi-analytical approaches of flux-tracking for the local dynamics of current density of AC SC wires immersed in a constant transverse magnetic field, from which all the macroscopical electromagnetic quantities of interest such as the magnetic field, magnetic moment, and power density of energy losses can be calculated. The observations reported for a rounded SC wire, define an adequate benchmark for the implementation of flux-tracking approaches in other $2 \mathrm{D}$ symmetries, such as SC strips, where the flux-front profile for isolated excitations can be formulated by exact geometrical expressions.
\end{abstract}

Index Terms-Type-II superconductors, Critical state, Magnetization of superconductors, AC-losses.

\section{INTRODUCTION}

$\mathbf{T}$ HE opportunities of technological applications that typeII superconducting wires provide include but are not limited to, electricity transmission lines, fault-currents limiters, magnetic resonance imaging (MRI), superconducting motors, generators, and magnet systems used in particle accelerators [1]. The usefulness of type-II superconductors in technological applications is related to the local electromagnetic response of the superconductor under transverse magnetic field and different conditions of transport current, with very low but no negligible AC-losses (power dissipation, E.J) [2][14]. For instance, due to the reduced use of space, high transport current capability, and nearly-zero resistive losses, type-II superconductors are expected to be extensively used in the designing of $\mathrm{DC}$ and $\mathrm{AC}$ power grids, where multiple

B. C. Robert and H. S. Ruiz are with the Department of Engineering and the Leicester Institute for Space \& Earth Observation Science of the University of Leicester, Leicester, LE17RH, U.K. (e-mail: bcr4@leicester.ac.uk dr.harold.ruiz@leicester.ac.uk

The authors acknowledge the use of the High Performance Computing Cluster Facilities (ALICE) provided by the University of Leicester, and to Dr. Antonio Badia-Majos from the Department of Condensed Matter Physics of the University of Zaragoza for his valuable comments and discussion. B. Robert thanks the Scholarship unit of the Niger Delta Development Commission for their financial support.

Manuscript submitted January 10, 2018. transmission lines could be sharing the same right of way, and therefore each of the lines could be subjected to external sources of magnetic field [15]. However, under the AC-DC conditions, the understanding of the electromagnetic response of AC superconducting wires results cumbersome [16][20]. This is due to the concomitant occurrence of magnetization and transport currents inside the superconducting material [21], impeding to obtain exact analytical solutions even for the case of infinitely long wires of cylindrical cross section. In fact, this geometry is of utter importance in applied superconductivity, as under certain physical conditions, it is still possible to find accurate analytical approaches which define a general benchmark for the calculation of AC-losses in type-II superconductors.

The understanding of the macroscopic electromagnetic behaviour of type-II superconducting wires can be captured by the numerical solution of the Maxwell equations under the framework of the critical state theory [22] and the socalled integral formulation [23], which is also known as the $\mathrm{J}$-formulation [24]. This formulation is of special relevance for superconducting systems where flux pinning and flux cutting effects interplay, which is a phenomena that can be observed when an external magnetic field aligns with the direction of the transport current in type-II superconductors [25]. Fortunately, the flux cutting phenomena can be avoided if for the application under question, the external magnetic field is always transverse to the direction of the profiles of current density inside the superconducting material.

This is indeed the case of the cylindrical superconducting wires considered in this study, which in a 2D approach obeys to the assumption that its radius $R$ is much smaller than its length, being this a fairly reasonable assumption in the analysis of power transmission lines. Thus, in this paper we present a comprehensive study on the effects of considering an AC transmission line of cylindrical cross section, made of a type-II superconductor with critical current density $J_{c}$, that is simultaneously exposed to a constant source (DC) of transverse magnetic field. Knowing the distribution of lines of magnetic field in this case, is also of special relevance for understanding the effects that introducing a superconducting wire inside of a MRI device could have, as the magnetization of the superconducting wire could lead to strong distortions on the image processing of magnetic resonance imaging.

\section{ELECTROMAGNETIC CONDITIONS AND FLUX FRONTS}

The numerical method and theory introduced in [23] has been exploited for the calculation of all the macroscopic electromagnetic quantities of interest, without the need to 


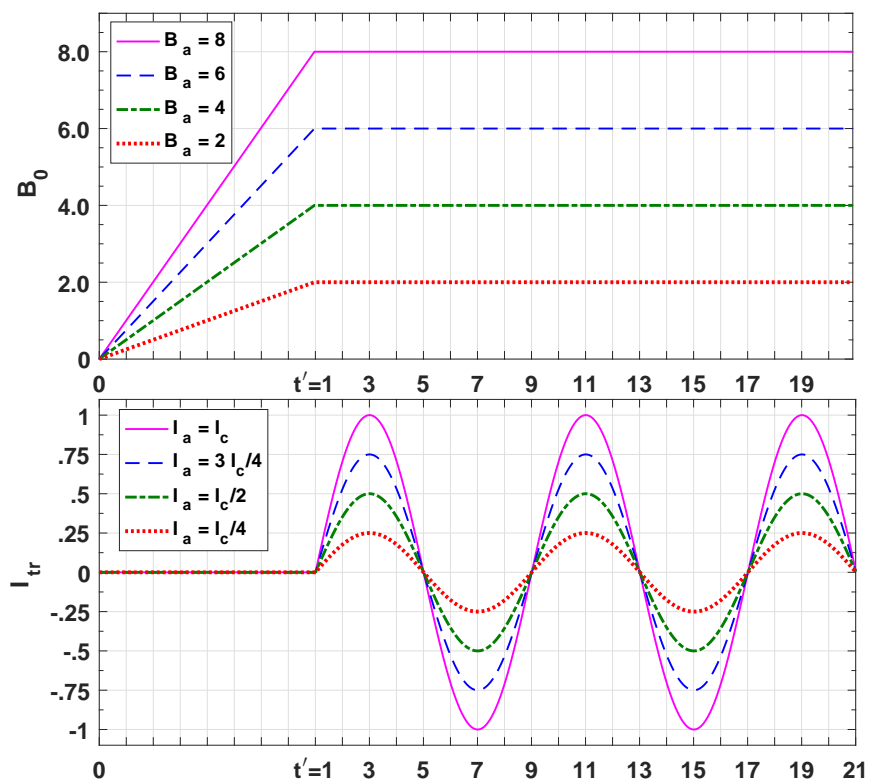

Fig. 1. (Color online) Time evolution of the electromagnetic excitations, longitudinal transport current $\left(I_{t r}\right)$ and transverse magnetic field $B_{0, y}$, that are applied to a cylindrical SC wire of radius $R$ lying over the plane $x y$ (see. Fig. 2. The amplitude of the AC transport current, $I_{a}$, is given in units of $J_{c} \pi R^{2}$, and $B$ units are $\left(\mu_{0} / 4 \pi\right) J_{c} R$.

include any boundary condition for the solution of partial differential equation systems, fundamental for the use of the so-called $\mathrm{H}$-formulation [26]. Time-dependent distributions for the flux front profiles of local current density, magnetic flux, and the cycles of magnetic moment are discussed for the case of study. In particular, in this section we present the formation of distinctive distributions of profiles of current density with mixed flux front profiles, where clear elliptical field-like distributions of current are embedded into welldefined rings of transport current, when the type-II SC wire is subjected to the experimental conditions depicted in Fig. 1 .

In Fig. 2 we present the local distribution of profiles of current density into the critical state regime, $J_{i}= \pm J_{c}$, where intricate shapes of the flux front profile can be identified. It can be noticed therein that at the time step $t^{\prime}$, labelled as $t=1$ in Figs. 1 \& 2 the AC transport current $\left(I_{t r}\right)$ is inceptively applied after a magnetization period which expands from the virgin state of the SC wire $\left(J_{i}=0 \in S C\right.$ domain $)$, i.e., from $t=0$ up to $t=t^{\prime}$ in Fig. 1, from which the applied magnetic field of intensity $B_{a}$ (in units of $\left(\mu_{0} / 4 \pi\right) J_{c} R$ ) is kept constant. Thus, immediately after $t^{\prime}$ the common fieldlike shape of the flux front profile observed in Fig. 211 is deformed by the injection of finite lines carrying the transport current whilst satisfying the condition, $\int J_{i} d x d y=I_{t r}$. As consequence, we have noticed a strong deformation of the axisymmetric orientation of the flux front profiles, which is evidently triggered by the competition between the injected transport current and the magnetisation currents inside the SC. Furthermore, this affects the geometrical centre of the flux free core, i.e., the regions without current density, causing it to displace towards the left side of the SC sample given the positive slope of the injected $I_{t r}$. In other words, the local

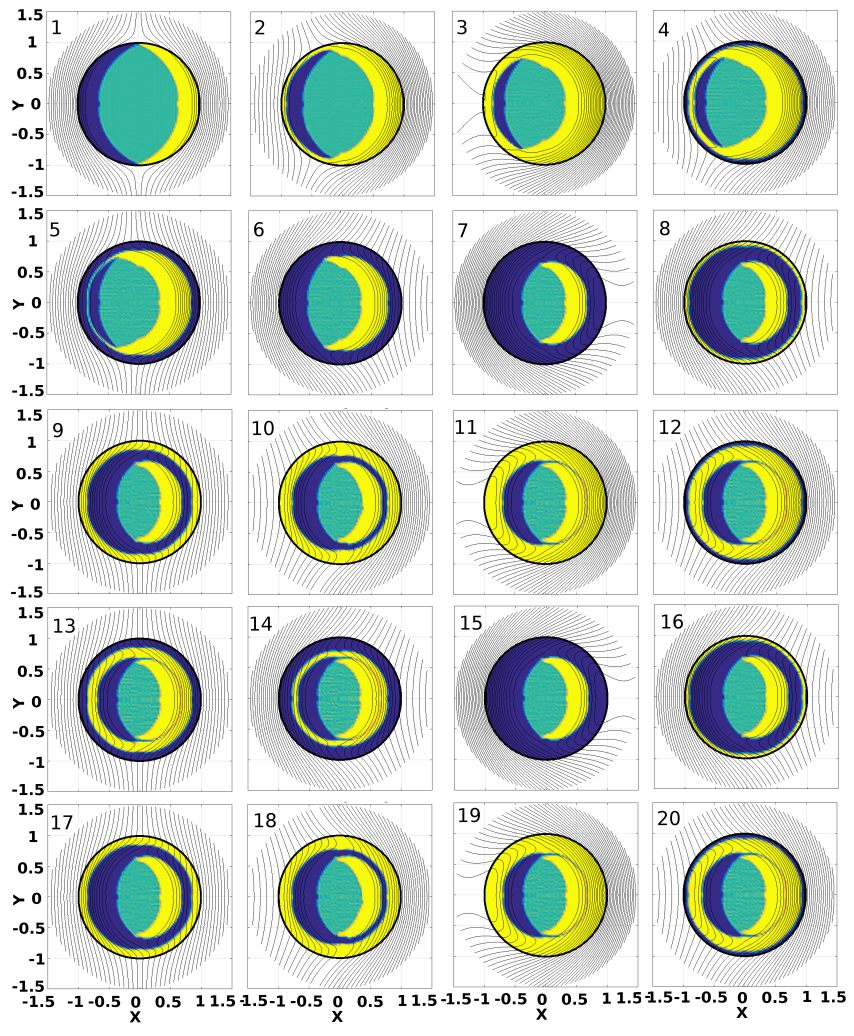

Fig. 2. (Color online) Flux front dynamics in a cylindrical superconducting wire of radius $R$ exposed to the electromagnetic excitations depicted in Fig. 1 with an AC $I_{t r}$ of moderate amplitude, $I_{a}=0.5 I_{c}$, and a DC magnetic field of amplitude $B_{a}=2$. Darker (blue) regions indicates critical state zones with $J_{i}=-J_{c}$, and the lighter (yellow) regions correspond to $J_{i}=+J_{c}$, otherwise $J_{i}=0$ (green zone) defines flux free conditions.

elements of transport current that are introduced to the SCsample consume the magnetization current lines at the negative abscissa of the cylindrical wire, and simultaneously exerts a Lorentz force pushing inwards the magnetization currents at the other side of the SC body. On the other hand, it is to be noticed that the symmetry of the ordinate elements of current is preserved, as the direction of the external magnetic field is not changed.

More noticeable is the fact that the phenomenon of consumption and displacement of the magnetization currents above described, can be observed only during the first three quarters of the cyclic transport current, i.e., up to the time step $t=7$, when the second peak of the transport current excitation is reached. Therefore, the deformation and changes on the size of the flux front profile of an AC superconducting wire exposed to a constant source of magnetic field, is an univocal characteristic of magnetic relaxation that can be straightforwardly observed in Fig. 2 Then, a cyclic hysteresis behaviour is achieved, as it can bee seen by comparing the distribution of profiles of current density at $t=7$ and $t=15$, establishing the regime for the adequate calculation of the $\mathrm{AC}$ (peak-to-peak) losses.

It can also be observed in Fig. 2 that after the magnetic stabilization period, not only the size and shape of the flux core does not change but also, it is possible to identify a circular 
flux front profile which is characteristic of transport current dynamics inside a type-II SC. The relevance of this finding is that current-like flux front profile in the hysteretic cyclic regime can be calculated by exact analytical methods [27], from which the boundary of the flux front corresponds to an axisymmetric circumference of radius:

$$
r=R \sqrt{1-\frac{I_{t r}}{I_{c}}},
$$

which confines the magnetization currents inside the transport current limit $I_{t r}=I_{a}$, i.e., $r_{a}=R \sqrt{1-I_{a} / I_{c}}$. Therefore, an inner secondary flux front profile can be conceived, which separately allows to identify the distribution of profiles of current density created by the external magnetic field with a centered semi-elliptical flux free core (see rows 3-5 in Fig. 2), which can be calculated from diverse semi-analytic approaches like the ones reported by Ashkin [28], Wagner [29], and Gömöry et al. [30], but by assuming a cylindrical wire of radius $r_{a}<R$. Thus, by using Gömöry's approach which can achieve precision levels of up to $99.9 \%$, a single semiempirical variable, $\varepsilon$, defining the semi-major axis $\left(\varepsilon r_{a}\right)$ of the elliptical flux front profile is introduced, such that the position of inner flux front for the field-like current distribution can be calculated in polar coordinates as:

$$
r_{\varepsilon}(\theta)=\frac{\varepsilon r_{a}}{\sqrt{\cos ^{2}(\theta)+\varepsilon^{2} \sin ^{2}(\theta)}},
$$

where $\epsilon$ can be tracked down from the magnetization measurements (see Fig. 3) with $M(\varepsilon)=-(2 / 3) H_{p^{\prime}}\left(1-\varepsilon^{2}\right)$, and $H_{p^{\prime}}=\mu_{0}^{-1} B_{p} \sqrt{1-I_{a} / I_{c}}$, the later defining the condition for full penetration of magnetic flux inside the SC wire, with $\varepsilon$ ranging from 0 , i.e., accounting for the disappearance of the flux free core, to 1, i.e., when the field-like flux front coincides with the current-like flux front, or inner surface of radius $r_{a}$ that limits the distribution of magnetization currents.

\section{Magnetization ChaRACTERISTiCs AND AC-Losses}

Following with the previous arguments, it is possible to state that as long as $I_{a}<I_{c}$, then $r_{a} \neq 0$ and consequently, the SC wire shows enough room to allocate profiles of magnetization current. Thus, is we assume that the SC wire is fully magnetized before applying the $\mathrm{AC}$ transport current $\left(B_{a}=8\right.$ in Fig. 11, the magnetization curve as a function of the transport current will show a monotonic increase of $M_{y}$ up to the saturation value $M_{p}=(2 / 3) J_{c} R^{3}$ (see Fig. 3 . Then, when the AC transport current is applied, the lines of injected transport current consume the lines of magnetization current, lowering the overall magnetization of the SC wire up to the complete disappearance of the magnetization signal when $I_{a}=I_{c}$. However, if $I_{a}<I_{c}$ and $B_{a} \neq 0$, we have already demonstrated that the full penetration of the magnetic field (or profiles of current density) is achieved at the field $H_{p^{\prime}}$ which is always smaller than $H_{p}$ by the factor $\sqrt{1-I_{a} / I_{c}}$. Thus, for applied magnetic fields greater than $H_{p^{\prime}}$, the flux free core which might be observed during the magnetic stabilization period, i.e, the first three quarters of the applied transport current (up to the time-step seven in Fig. 1 and Fig. 2p eventually disappears, leading to the stabilization of
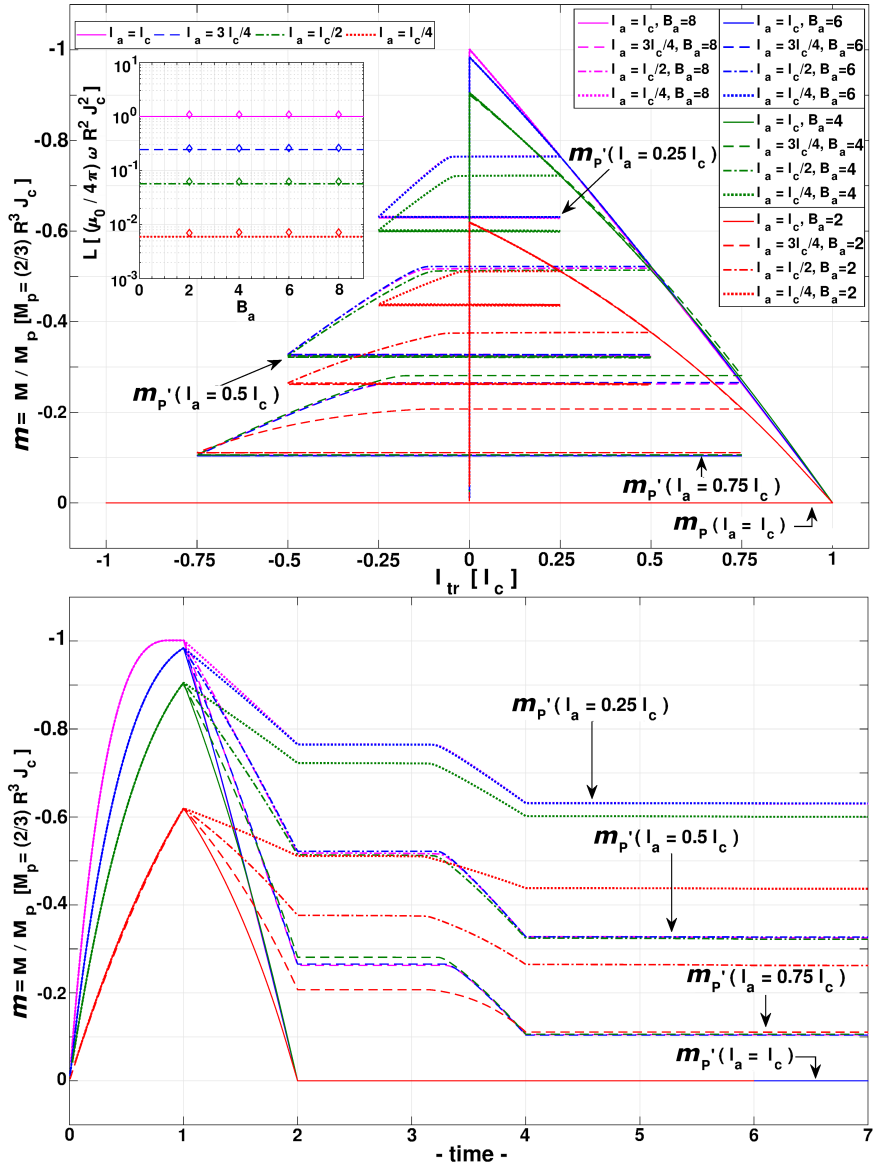

Fig. 3. (Color online) Dimensionless magnetization curves $\left(m=M_{y} / M_{p}\right)$ as function of the $\mathrm{AC}$ transport current $I_{t r}$ and the temporal/experimental process displayed in Fig. $11 M_{p}=2 J_{c} R^{3} / 3$ is the analytical solution for the magnetic moment of the sample when it is fully penetrated by a field $B_{p}=8$ with zero transport current. Recall that units for $B_{a}$ are $\left(\mu_{0} / 4 \pi\right) J_{c} R, R$ is the radius of the SC wire, and $I_{t r}$ is given in units of $I_{c}$. In the upper left inset, our numerical results (solid symbols) for the calculation of the monotonous hysteresis losses per cycle of the transport current are presented. Therein, the solid lines correspond to the analytical solution described by Eq. $4 L$ is shown in units of $\left(\mu_{0} / 4 \pi\right) \omega R^{2} J_{c}^{2}$ per cycles of frequency $\omega$.

the finite magnetic moments inside the circumference of radius $r_{a}$. Therefore, the maximum magnetization of a premagnetized rounded SC wire with AC transport current can be calculated from the semi-analytical approach $M_{p}=-(2 / 3) J_{c} r_{a}^{3}$, which can be written as a function of $I_{a}$ as follows,

$$
M_{p}^{\prime}\left(I_{a}\right)=-\frac{2}{3} J_{c} R^{3}\left(1-\frac{I_{a}}{I_{c}}\right)^{3 / 2} .
$$

This result implies, for instance, that for $I_{a}=0.5$ the maximum dimensionless magnetization that the $\mathrm{SC}$ wire can achieve is $m_{p}^{\prime}=M_{p}^{\prime} / M_{p}=-0.3535$, in good agreement with our numerical results (see Fig. 3). Furthermore, $m_{p}^{\prime}$ can only be reached for $B_{a}>B_{p} \sqrt{1-0.5} \approx 5.65$, and although the difference between the resulting magnetic moment for $B_{a}>4$ and $I_{a}$ is almost negligible, for lower magnetic fields showing a wide flux-free core (Fig. 2) a smaller magnetization plateau, $m^{\prime}\left(I_{a}=0.5, B_{a}=2\right) \approx-0.262$, can be observed. Therefore, for applied magnetic fields with $B_{a}<B_{p} \sqrt{1-I_{a} / I_{c}}$, the existence of a flux free core inside a $\mathrm{SC}$ rounded wire subjected 
to AC transport current and DC magnetic field can be seen from the macroscopic measurement of the magnetic moment of the wire, when $|m|<\left|m_{p}^{\prime}\right|$. Otherwise, for the experimental conditions depicted in Fig. 1 , and after the cycle of magneticstabilization, a constant magnetization plateau $\left(m_{p}^{\prime}\right)$ which does not depend on the time-dynamics of the transport current is to be observed.

Finally, concerning to the density of power losses $(\mathbf{E} \cdot \mathbf{J})$ during the cycles of transport current, it is clear that although the external magnetic field is kept constant during this period, the first excitation cycle defines a period of magnetic stabilization where the profiles of current density that contribute to the magnetization are regrouped into the $r_{a}$ boundary, showing a constant field-like profile afterwards. In other words, the so-called AC-losses or cyclic hysteretic losses under these conditions, must be calculated fro the second cycle of $I_{t r}$. Moreover, as the electric field vector can be defined in terms of the magnetic vector potential as $\mathbf{E}=-\partial_{t} \mathbf{A}-C_{t} \hat{\mathbf{u}}_{k}$, where thanks to the symmetry of the problem $\nabla \phi=C_{t} \hat{\mathbf{u}}_{k}$ becomes an integration constant, and the contributions of the external magnetic field and the induced/injected transport current can be grouped into separate vector potentials for the elements of current $J_{i}\left(\mathbf{r}_{i}\right)$, i.e., $\mathbf{A}_{\text {ext }}\left(\mathbf{r}_{i}\right)=\mathbf{B}_{0} \times \mathbf{r}_{i}$ and, $A_{\text {self }}=$ $\mu_{0} \pi J_{i} /(4 \pi)$ for the self inductance contributions, and $A_{m u t}=$ $-\mu_{0} J_{i} \ln \left(r_{i j}^{2}\right) /(4 \pi)$ for the mutual inductance contributions between the $i \rightarrow j$ finite elements of current of circular crosssection [2], [31]. Therefore, with $A_{\text {ind }}=A_{\text {self }}+A_{m u t}$, it is clear that only the induced elements of current contribute to the AC-losses, and it has been demonstrated that $J_{i}$ changes only inside the flux front boundaries for current-like distributions, $r_{a}<r<R$. Thus, although in our numerical calculations we have observed a very small increase in the integration constant $C_{t}$ as consequence of the magnetic stabilization period, we have found that (see top-inset in Fig. 3), for a rounded SC wire under constant transverse magnetic field and $\mathrm{AC}$ transport current, the AC-losses per unit time can be calculated by the analytical solution [27],

$$
L=\omega \frac{\mu_{0}}{2 \pi} R^{2} J_{c}^{2}\left[j-\frac{j^{2}}{2}+(1-j) \operatorname{Ln}(1-j)\right] .
$$

with $j=J_{t r} / J_{c}$, and $J_{t r}=I_{t r} /\left(\pi R^{2}\right)$.

\section{CONCLUSION}

In this paper, the concomitant action of a transverse DC magnetic field applied to a rounded superconducting wire of radius $R$ and critical current density $J_{c}$, carrying an alternating transport current of intensity $I_{a}$ (see Fig. 11 has been studied.

Based on the numerical results which have been obtained under the well-known framework of the critical state theory and the integral formulation introduced in [23], we have demonstrated that under the experimental conditions aforementioned, it is possible to attain a set of well established semi analytical expressions to track the evolution of the flux front profile, after a period of magnetic stabilization which spans during the first cycle of oscillation of $I_{t r}(t)$. During this period of time, a marked redistribution of the lines of magnetization current is observed due to the Lorentz force added by the insertion of finite lines of transport current density which satisfy the condition $\int J_{i} d \Omega=I_{t r}$, being $\Omega$ the cross-section area of the SC wire. The resulting distribution of profiles of current density $J_{i}= \pm J_{c}$ or 0 that is observed during this cycle follows a cumbersome dynamics which precludes a semianalytical description of the flux front profile. However, once the SC wire reaches the cyclic monotonic behaviour, we have noticed the occurrence of two flux front boundaries that define the limits for field-like and current-like distributions of current density. On the one hand, the dynamics of the flux front profile during the monotonic regime is confined to region of the space limited by the condition $r_{a}<r<R$, with $r_{a}=R \sqrt{1-I_{a} / I_{c}}$, where circular "current-like" profiles of current density occurs. On the other hand, the inner limit $r_{a}$ defines the outer boundary of semi-elliptical "field-like" profiles of current density contributing to the overall (constant) magnetization of the superconducting wire. Therefore, we have found that not only the distribution of profiles of current density could be conceived without the need of recurring to the somehow cumbersome numerical algorithms, but also the macroscopic quantities that can be experimentally extracted such as the maximum magnetic moment of the SC wire, $M_{p}$, and the AC-losses, $L$, can both be accurately determined by the simplification of considering firstly "current-like" flux front profiles for the dynamics of $I_{t r}(t)$, and secondly the embedding of a constant "field-like" distribution. Given the generality of our approach, and consequently of our observations, the results here presented can be straightforwardly extended to SC strips with elliptical cross-section [30], and any other 2D symmetry where semi-analytical approaches of flux front-tracking are known.

\section{REFERENCES}

[1] S. S. Kalsi, Applications of High Temperature Superconductors to Electric Power Equipment. Wiley-IEEE Press, 2011.

[2] H. S. Ruiz, A. Badía-Majós, Y. A. Genenko, H. Rauh, and S. V. Yampolskii, "Superconducting wire subject to synchronous oscillating excitations: Power dissipation, magnetic response, and low-pass filtering," Applied Physics Letters, vol. 100, no. 11, p. 112602, 2012. [Online]. Available: http://dx.doi.org/10.1063/1.3693614

[3] J. Ogawa, M. Shiokawa, M. Ciszek, and O. Tsukamoto, "Ac losses in ybco coated conductors carrying ac transport currents in perpendicular ac external magnetic field," IEEE Transactions on Applied Superconductivity, vol. 13, no. 2, pp. 1735-1738, June 2003.

[4] J. Ogawa, S. Fukui, T. Oka, T. Sato, H. Kojima, M. Shibayama, and M. Egawa, "Influence of dc offset transport current on ac loss characteristics in hts tapes," IEEE Transactions on Applied Superconductivity, vol. 21, no. 3, pp. 3325-3328, June 2011.

[5] F. G'om'ory, M. Vojenčiak, E. Pardo, M. Solovyov, and J. Šouc, "Ac losses in coated conductors," Superconductor Science and Technology, vol. 23, no. 3, p. 034012, 2010. [Online]. Available: http://stacks.iop.org/0953-2048/23/i=3/a=034012

[6] M. Vojenčiak, J. Souc, J. M. Ceballos, F. G'om'ory, B. Klinčok, E. Pardo, and F. Grilli, "Study of ac loss in bi-2223/ag tape under the simultaneous action of ac transport current and ac magnetic field shifted in phase," Superconductor Science and Technology, vol. 19, no. 4, p. 397, 2006. [Online]. Available: http://stacks.iop.org/0953-2048/19/i=4/a=026

[7] E. Pardo, F. G'om'ory, J. Souc, and J. M. Ceballos, "Current distribution and ac loss for a superconducting rectangular strip with in-phase alternating current and applied field," Superconductor Science and Technology, vol. 20, no. 4, p. 351, 2007. [Online]. Available: http://stacks.iop.org/0953-2048/20/i=4/a=009

[8] Z. Hong, L. Ye, M. Majoros, A. M. Campbell, and T. A. Coombs, "Numerical estimation of ac loss in mgb2 wires in selffield condition," Journal of Superconductivity and Novel Magnetism, 
vol. 21, no. 3, pp. 205-211, Apr 2008. [Online]. Available: https://doi.org/10.1007/s10948-008-0319-y

[9] N. Nibbio and S. Stavrev, "Effect of the geometry of hts on ac loss by using finite element method simulation with b-dependent e-j power law," IEEE Transactions on Applied Superconductivity, vol. 11, no. 1, pp. 2627-2630, Mar 2001.

[10] N. Amemiya and Y. Ohta, "Mode of magnetic flux penetration into high tc superconductors with various cross-sectional shape and their ac loss characteristics," Physica C: Superconductivity, vol. 357, pp. 1134 - 1143, 2001. [Online]. Available: http: //www.sciencedirect.com/science/article/pii/S0921453401005408

[11] B. ten Haken, J.-J. Rabbers, and H. H. ten Kate, "Magnetization and ac loss in a superconductor with an elliptical cross-section and arbitrary aspect ratio," Physica C: Superconductivity, vol. 377, no. 1, pp. 156 - 164, 2002. [Online]. Available: http: //www.sciencedirect.com/science/article/pii/S0921453401011947

[12] J. L. Giordano, J. Luzuriaga, A. Badía-Majós, G. Nieva, and I. RuízTagle, "Magnetization collapse in polycrystalline ybco under transport current cycles," Superconductor Science and Technology, vol. 19, no. 4, p. 385, 2006. [Online]. Available: http://stacks.iop.org/0953-2048/19/i= $4 / \mathrm{a}=024$

[13] F. Grilli, E. Pardo, A. Stenvall, D. N. Nguyen, W. Yuan, and F. G'om'ory, "Computation of losses in hts under the action of varying magnetic fields and currents," IEEE Transactions on Applied Superconductivity, vol. 24, no. 1, pp. 78-110, Feb 2014.

[14] A. Stenvall, V. Lahtinen, and M. Lyly, "An h -formulation-based three-dimensional hysteresis loss modelling tool in a simulation including time varying applied field and transport current: the fundamental problem and its solution," Superconductor Science and Technology, vol. 27, no. 10, p. 104004, 2014. [Online]. Available: http://stacks.iop.org/0953-2048/27/i=10/a=104004

[15] H. Thomas, A. Marian, A. Chervyakov, S. St'uckrad, D. Salmieri, and C. Rubbia, "Superconducting transmission lines - sustainable electric energy transfer with higher public acceptance," Renewable and Sustainable Energy Reviews, vol. 55, no. Supplement C, pp. 59 - 72, 2016. [Online]. Available: http://www.sciencedirect.com/science/article/ pii/S136403211501120X

[16] W. J. Carr, AC loss and macroscopic theory of superconductors. Taylor \& Francis, 2001.

[17] G. P. Mikitik, Y. Mawatari, A. T. S. Wan, and F. Sirois, "Analytical methods and formulas for modeling high temperature superconductors," IEEE Transactions on Applied Superconductivity, vol. 23, no. 2, pp. 8001920-8001920, April 2013.

[18] F. Sirois and F. Grilli, "Potential and limits of numerical modelling for supporting the development of hts devices," Superconductor Science and Technology, vol. 28, no. 4, p. 043002, 2015. [Online]. Available: http://stacks.iop.org/0953-2048/28/i=4/a=043002

[19] V. Lahtinen, M. Lyly, A. Stenvall, and T. Tarhasaari, "Comparison of three eddy current formulations for superconductor hysteresis loss modelling," Superconductor Science and Technology, vol. 25, no. 11, p. 115001, 2012. [Online]. Available: http://stacks.iop.org/0953-2048/ $25 / \mathrm{i}=11 / \mathrm{a}=115001$

[20] S. T. Ranecky, H. Watanabe, J. Ogawa, T. Oka, D. G'olden, L. Alff, and Y. A. Genenko, "Experimental and theoretical study of ac losses in variable asymmetrical magnetic environments," Superconductor Science and Technology, vol. 30, no. 8, p. 085005, 2017. [Online]. Available: http://stacks.iop.org/0953-2048/30/i=8/a=085005

[21] H. S. Ruiz and A. Badía-Majós, "Exotic magnetic response of superconducting wires subject to synchronous and asynchronous oscillating excitations," Journal of Applied Physics, vol. 113, no. 19, p. 193906, 2013. [Online]. Available: http://dx.doi.org/10.1063/1.4804931

[22] C. P. Bean, "Magnetization of high-field superconductors," Rev. Mod. Phys., vol. 36, pp. 31-39, Jan 1964. [Online]. Available: https://link.aps.org/doi/10.1103/RevModPhys.36.31

[23] A. Badía-Majós, C. López, and H. S. Ruiz, "General critical states in type-ii superconductors," Phys. Rev. B, vol. 80, p. 144509, Oct 2009. [Online]. Available: https://link.aps.org/doi/10.1103/PhysRevB. 80.144509

[24] H. S. Ruiz and A. Badía-Majós, "Smooth double critical state theory for type-ii superconductors," Superconductor Science and Technology, vol. 23, no. 10, p. 105007, 2010. [Online]. Available: http://stacks.iop.org/0953-2048/23/i=10/a=105007

[25] H. S. Ruiz, C. López, and A. Badía-Majós, "Inversion mechanism for the transport current in type-ii superconductors," Phys. Rev. $B$, vol. 83, p. 014506, Jan 2011. [Online]. Available: https: ///ink.aps.org/doi/10.1103/PhysRevB.83.014506
[26] M. Baghdadi, H. S. Ruiz, and T. A. Coombs, "Crossed-magneticfield experiments on stacked second generation superconducting tapes: Reduction of the demagnetization effects," Applied Physics Letters, vol. 104, no. 23, p. 232602, 2014. [Online]. Available: http://dx.doi.org/10.1063/1.4879263

[27] A. V. Gurevich, R. G. Mints, and A. L. Rakhmanov, Physics of Composite Superconductors. Begell House, New York, 1997.

[28] M. Ashkin, "Flux distribution and hysteresis loss in a round superconducting wire for the complete range of flux penetration," Journal of Applied Physics, vol. 50, no. 11, pp. 7060-7066, 1979. [Online]. Available: http://dx.doi.org/10.1063/1.325866

[29] M. Ashkin and G. R. Wagner, "Comparison of calculated and measured hysteresis loss in multifilamentary superconducting wire," Journal of Applied Physics, vol. 60, no. 7, pp. 2477-2481, 1986. [Online]. Available: http://dx.doi.org/10.1063/1.337161

[30] F. G'om'ory, R. Tebano, A. Sanchez, E. Pardo, C. Navau, I. Husek, F. Strycek, and P. Kovac, "Current profiles and ac losses of a superconducting strip with an elliptic cross-section in a perpendicular magnetic field," Superconductor Science and Technology, vol. 15, no. 9, p. 1311, 2002. [Online]. Available: http://stacks.iop.org/0953-2048/15/i=9/a=306

[31] A. Badía and C. López, "Vector magnetic hysteresis of hard superconductors," Phys. Rev. B, vol. 65, p. 104514, Feb 2002. [Online]. Available: https://link.aps.org/doi/10.1103/PhysRevB.65.104514 